\title{
MISCDLANEA
}

PRIMERAS JORNADAS CIENTÍfICAS DE ESTUDIANTES DE
ENFERMERÍA. FACULTAD CIENCIAS DE LA SALUD.
UNIVERSIDAD DE ALICANTE.

\section{REVISIÓN SOBRE LA ENFERMERÍA DE ENLACE}

Morote Segarra, A.

Estudiante Facultad de Ciencias de la Salud. Universidad de Alicante. 


\section{INTRODUCCIÓN Y OBJETIVO:}

Para responder al incremento de la patología crónica en nuestra sociedad, es imprescindible el desarrollo de nuevas estrategias organizativas y asistenciales centradas en el paciente; orientadas al autocuidado y coordinación entre distintos niveles asistenciales.

En la literatura se encuentran diversas definiciones sobre gestión de casos. Todas tratan de presentarla como una herramienta utilizada por las enfermeras para dar continuidad de cuidados a la persona; herramienta que sigue una metodología científica de valoración, diagnóstico, objetivo, intervención y evolución, y que permite y exige el trabajo en equipo, así como la coordinación entre diferentes actores.

Se aborda aquí un análisis de publicaciones en España, comprendidas entre enero de 2009 y junio de 2012 con el objetivo de presentar una revisión bibliográfica.

\section{MATERIAL Y MÉTODO:}

Se incluyeron los siguientes descriptores en la base de datos encuentr@: "gestión casos", "relación de ayuda", "continuidad de cuidados" y "enfermería enlace".

La búsqueda inicial arrojó un total de 243 artículos, de los cuales se seleccionaron aquellos que describiesen experiencias de trabajo en continuidad de cuidados, gestión de casos o enfermería de enlace y que incluyesen en sus descriptores la palabra clave "relación de ayuda". Se excluyeron del posterior análisis aquellos artículos que no estaban publicados en revistas científicas (capítulos de libro, manuales de procedimientos, etc.) y aquellos cuyo eje central no correspondía propiamente con los intereses de la revisión, en los que se aludía de manera superficial a la gestión de casos y/o a la relación de ayuda.

Finalmente se revisaron y clasificaron un total de 16 artículos. 


\section{RESULTADOS:}

De los 16 artículos, 7 siguen una metodología cualitativa-descriptiva, 7 se basan en revisiones bibliográficas, uno sigue una metodología cuantitativa y otro una metodología descriptiva-exploratoria (Fig.1).

Se ha hecho una clasificación según los diagnósticos médicos de los artículos revisados. A pesar de la heterogeneidad de los diagnósticos encontrados, parece evidente que son los pacientes con patología crónica los más beneficiados de la gestión de casos (Fig. 2).

La Figura 3 muestra la distribución de los artículos revisados por año de publicación. Dentro del período de tiempo revisado (2009-2012) encontramos un mayor número de publicaciones sobre gestión de casos y relación de ayuda entre los años 2010 y 2011 (Fig. 3).

Tal y como se aprecia en la Figura 4, del total de artículos revisados, diez aparecen publicados en la revista Metas de Enfermería, tres en Educare21, dos en Enfermería Familiar y Comunitaria y uno en Ciberevista (Fig. 4).

Figura 1: Tipo de estudio (metodología de los artículos revisados):

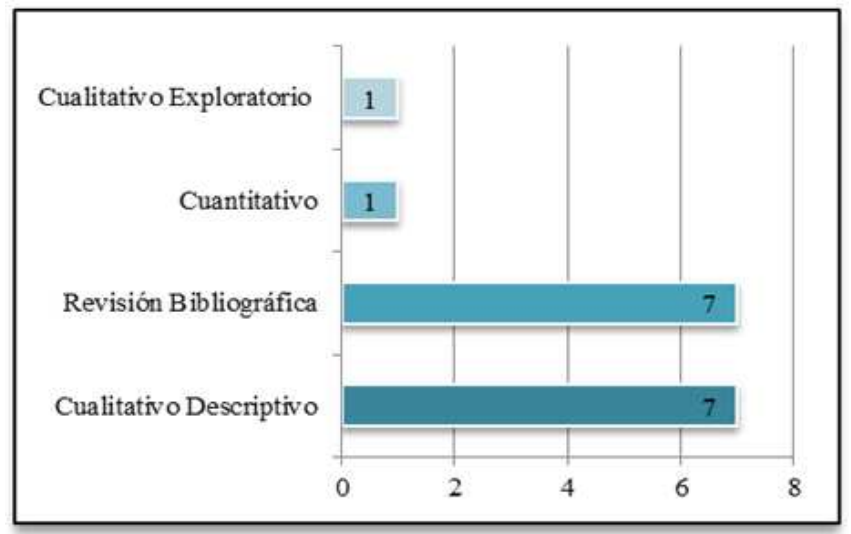


Figura 2: Diagnósticos de las muestras objeto de estudio:

\begin{tabular}{lc}
\hline Diagnóstico Médico & Número Artículos \\
\hline Enfermedad neurológica & 2 \\
\hline Insuficiencia cardíaca & 1 \\
\hline Neuropatía hipomielinizante congénita & 1 \\
\hline Enfermedad terminal & 1 \\
\hline Embarazo no evolutivo & 1 \\
\hline Síndrome Fatiga Crónica & 1 \\
\hline Fractura de cadera & 1 \\
\hline Transplante médula & 1 \\
\hline Otros N/E & 7 \\
\hline
\end{tabular}

Figura 3: Distribución por años:

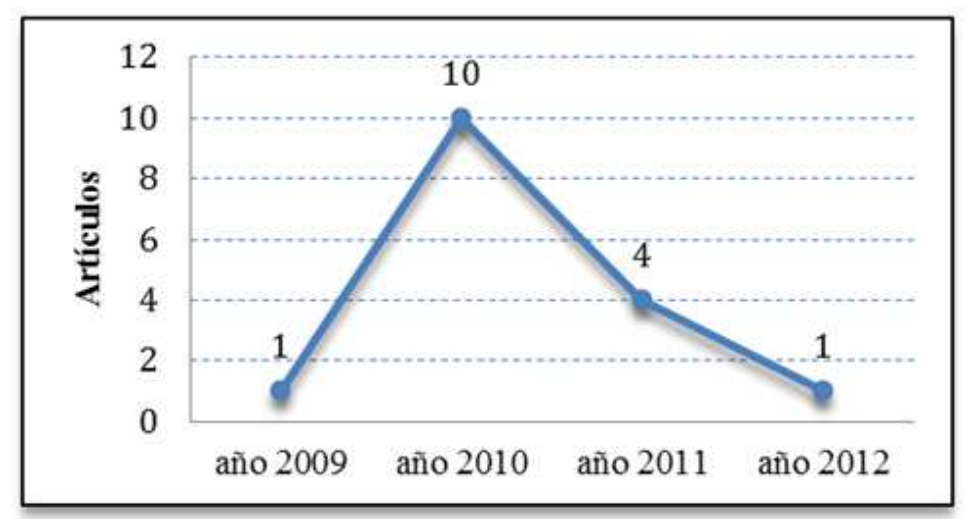

Figura 4: Distribución por revista de publicación:

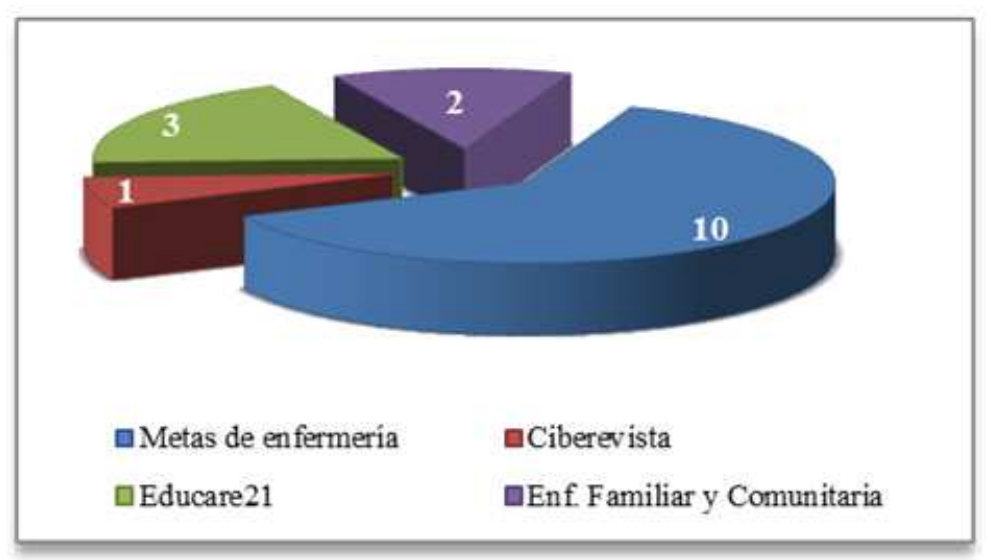




\section{CONCLUSIONES / DISCUSIÓN:}

Ha habido un avance en cuanto a publicaciones específicas en la continuidad de cuidados, gestión de casos y relación de ayuda (encontrando un mayor número entre 2010-2011). Aun así existe poca bibliografía específica para estos términos.

El nuevo rol de la enfermera gestora de casos, garante de la continuidad de cuidados, redunda en una mayor satisfacción de los profesionales sanitarios y de los pacientes, así como en una contención de costes, mejora de los indicadores de calidad asistencial y los resultados de salud en general.

Establecer una relación clínica basada en una comunicación eficaz con el paciente y/o cuidadores, en la confianza, el respeto y la empatía entre el personal de enfermería y el paciente, es imprescindible para garantizar una atención integral y continuada. La gestión de casos ha demostrado tener una influencia positiva sobre el autocuidado y la adherencia al régimen terapéutico.

Es necesario que continúen las investigaciones que aborden de manera crítica y estandarizada la gestión de casos y la continuidad de cuidados para poder protocolizarlos e incluirlos en los planes actuales y futuros de cuidados.

\section{AGRADECIMIENTOS:}

Agradezco la inestimable colaboración en la revisión literaria que ha permitido la realización de este póster a: Ramón Vicedo Cano; Elisa Marín Sánchez ; Ángela Pérez Sánchez; Ma Carmen Martínez González; Sin olvidar la ayuda de María Cabello Ganuza. 


\section{BIBLIOGRAFÍA}

- Calero Romero MR (2012). Percepción del paciente sometido a trasplante de médula ósea sobre la continuidad de los cuidados. Educare21; 10(3).

- Díaz Sánchez V et al. (2010). La construcción social del duelo en el aborto espontáneo. Metas de Enferm; 13(6): 25-32.

- Espina Boixo MA et al. (2011). Continuidad de cuidados desde el enfermero de emergencias hacia la enfermera de CI de pacientes con SCACEST. Metas de Enferm; 14(2): 60-65.

- Fernández Morenatti MA (2010). Experiencia de trabajo en un proceso de hospitalización domiciliaria. Metas de Enferm; 13(1): 58-61.

- Fontanet Cornudella G et al. (2010). La Enfermera virtual, un portal de educación y promoción para la salud. Metas de Enferm; 13(7): 50-54.

- García Pérez E (2009). La gestión de casos en la Clasificación de Intervenciones de Enfermería. Metas de Enferm; 12(4): 50-57.

- García Verde I et al. (2010). Problemas enfermeros en pacientes en situación terminal: caminando hacia la gestión de casos. Metas de Enferm; 13(3): 26-31.

- Hanzeliková Pogrányivá A y Pomares Martínez D (2010). Plan de cuidados estandarizado: fractura de cadera en pacientes geriátricos. Educare 21; 3(8).

- Jiménez Fernández JC y Cerrillo Martín D (2010) Registros de Enfermería: un espejo del trabajo asistencial. Metas de Enferm; 13(6): 8-11.

- Morales Asencio JM (2010). El liderazgo de la atención a personas con enfermedades crónicas complejas. RidEC; 3(2): 97-105.

- Padilla Just MN et al. (2011). Seguimiento telefónico enfermero a pacientes con insuficiencia cardíaca. Metas de Enferm; 14(3): 22-26.

- Ramírez Sánchez A (2011). Síndrome de fatiga crónica en el adulto: atención enfermera. Educare21; 77.

- Saavedra Llobregat MD et al. (2010) Cuidadores familiares. Aprendiendo a vivir. RIdEC; 3(2): 84-90.

- Sanz Peces EM et al. (2010). Atención enfermera a pacientes con enfermedades neurodegenerativas en fase avanzada. Metas de Enferm; 13(9): 29-32.

- Vázquez Calatayud M (2010). La vulnerabilidad y su relación con el cuidado. Metas de Enferm; 13(3): 72-74.

- Vergara Santos S (2011). Implantación de plan de cuidados para pacientes pluripatológicos de la UGC de Medicina Interna-B del Hospital Universitario "Virgen Macarena". Ciberevista; 19. 


\section{Revisión sobre la enfermería de enlace

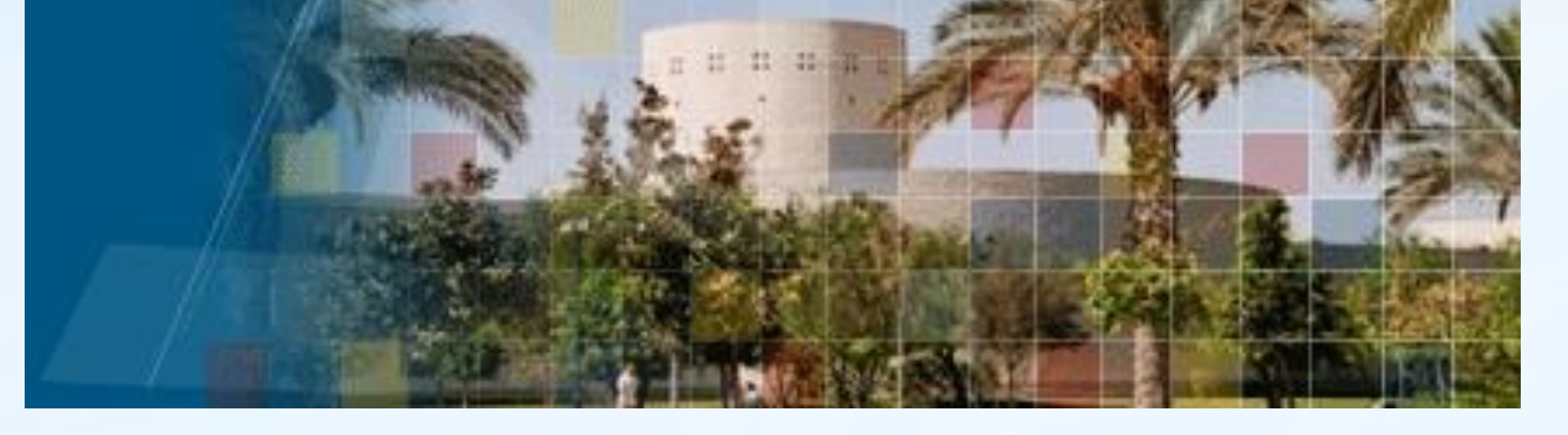 \\ Morote Segarra A. ${ }^{1}$ \\ Estudiante Facultad de Ciencias de la Salud, Universidad de Alicante}

\section{Introducción y objetivo}

Para responder al incremento de la patología crónica en nuestra sociedad, es imprescindible el desarrollo de nuevas estrategias organizativas y asistenciales centradas en el paciente; orientadas al autocuidado y coordinación entre distintos niveles asistenciales.

En la literatura se encuentran diversas definiciones sobre gestión de casos. Todas tratan de presentarla como una herramienta utilizada por las enfermeras para dar continuidad de cuidados a la persona; herramienta que sigue una metodología científica de valoración, diagnóstico, objetivo, intervención y evolución, y que permite y exige el trabajo en equipo, así como la coordinación entre diferentes actores.

Se aborda aquí un análisis de publicaciones en España, comprendidas entre enero de 2009 y junio de 2012) con el objetivo de presentar una revisión bibliográfica.
De los 16 artículos, 7 siguen una metodología cualitativadescriptiva, 7 se basan en revisiones bibliográficas, uno sigue una metodología cuantitativa y otro una metodología descriptiva-exploratoria (Fig.1).

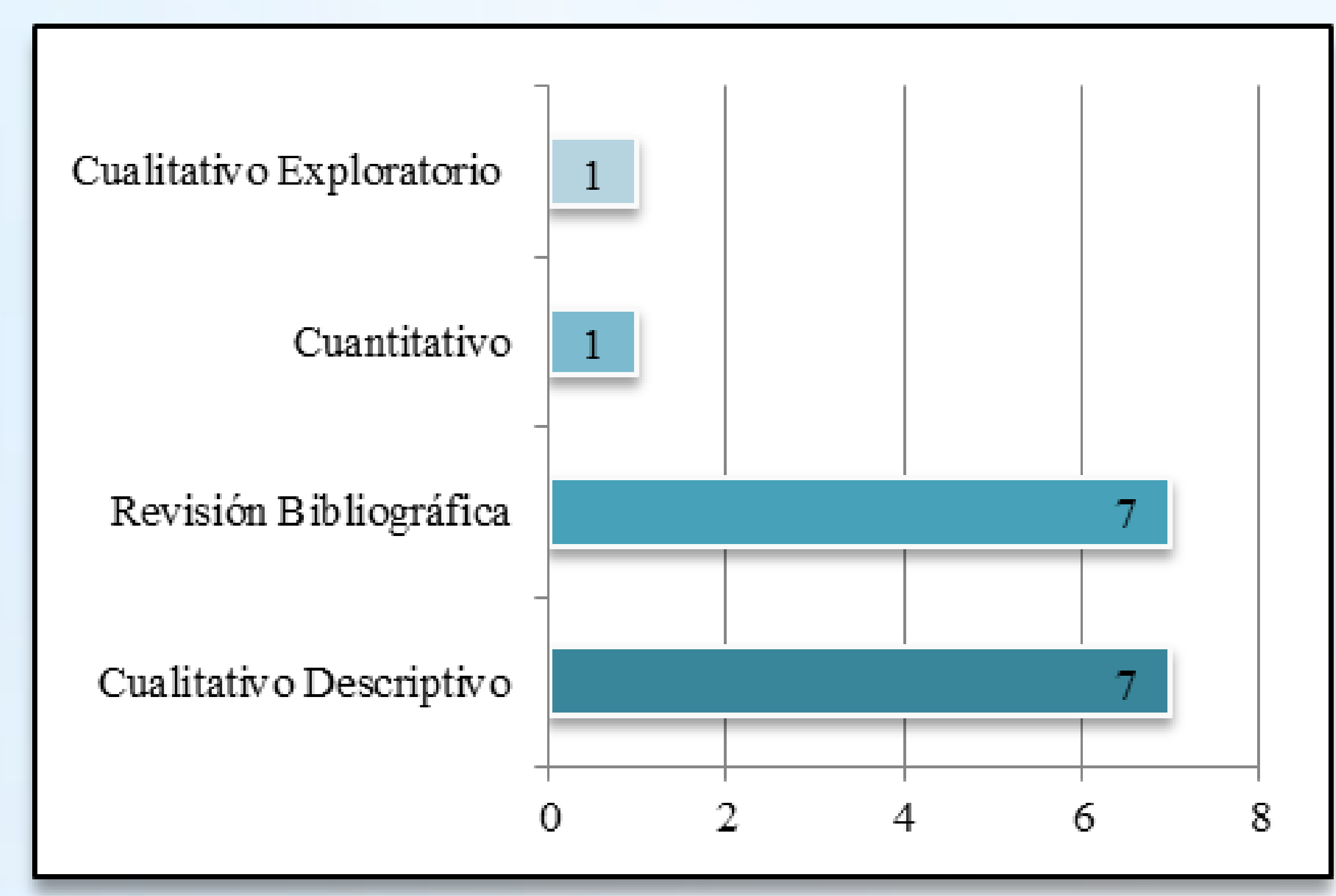

Figura 1: Tipo de estudio (metodología de los artículos revisados).

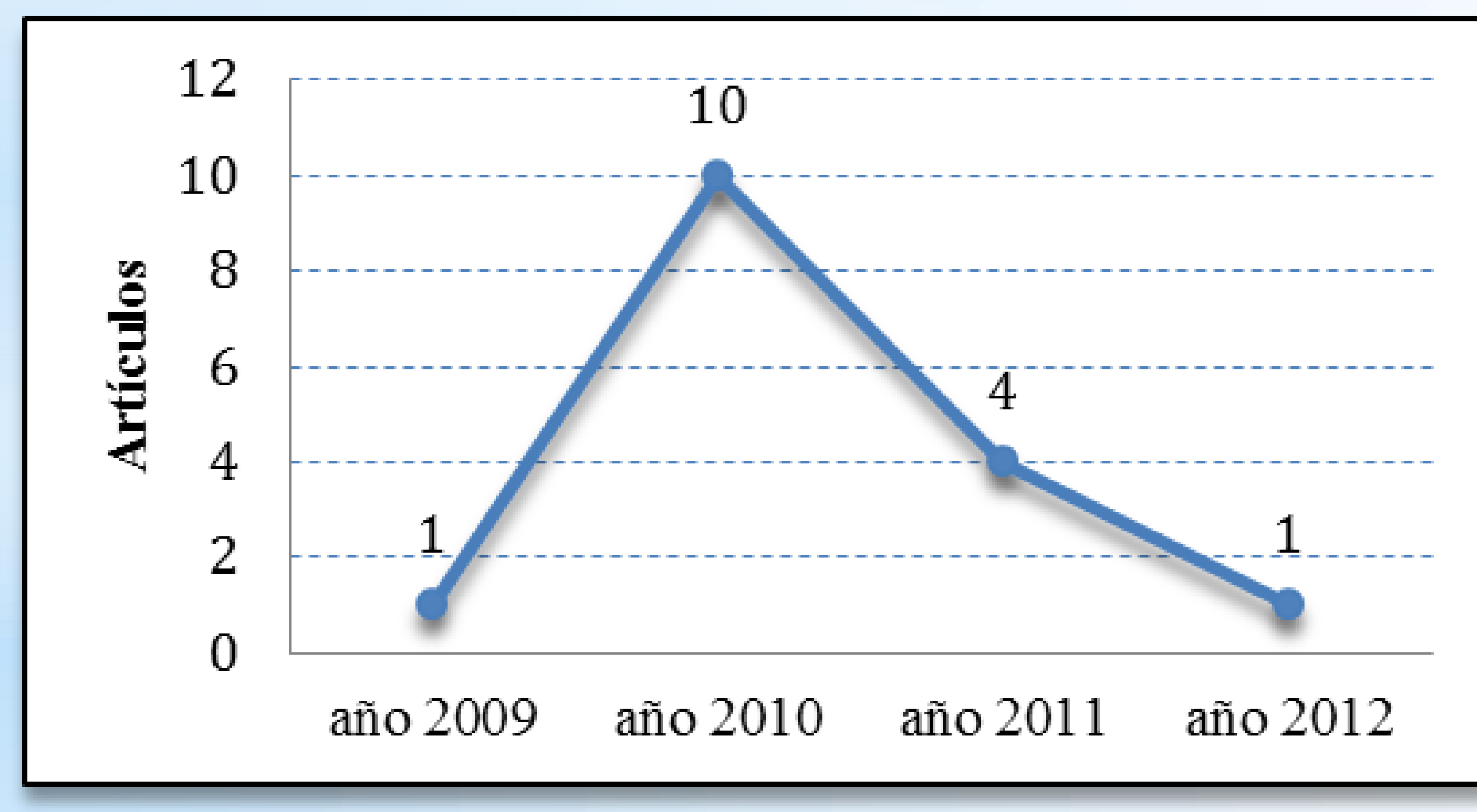

Figura 3: Distribución por años.
Se ha hecho una clasificación según los diagnósticos médicos de los artículos revisados. A pesar de la heterogeneidad de los diagnósticos encontrados, parece evidente que son los pacientes con patología crónica los más beneficiados de la gestión de casos (Fig. 2).

Figura 2: Diagnósticos de las muestras objeto de estudio.

\section{Diagnóstico Médico}

Enfermedad neurológica

Insuficiencia cardíaca

Neuropatía hipomielinizante congénita

Enfermedad terminal

Embarazo no evolutivo

Síndrome Fatiga Crónica

Fractura de cadera

Transplante médula

Otros N/E
Número Artículos

2

1

1

1

7

Tal y como se aprecia en la Figura 4, del total de artículos revisados, diez aparecen publicados en la revista Metas de Enfermería, tres en Educare21, dos en Enfermería Familiar y Comunitaria y uno en Ciberevista.

La Figura 3 muestra la distribución de los artículos revisados por año de publicación. Dentro del período de tiempo revisado (2009-2012) encontramos un mayor número de publicaciones sobre gestión de casos y relación de ayuda entre los años 2010 y 2011.

Figura 4: Distribución por revista de

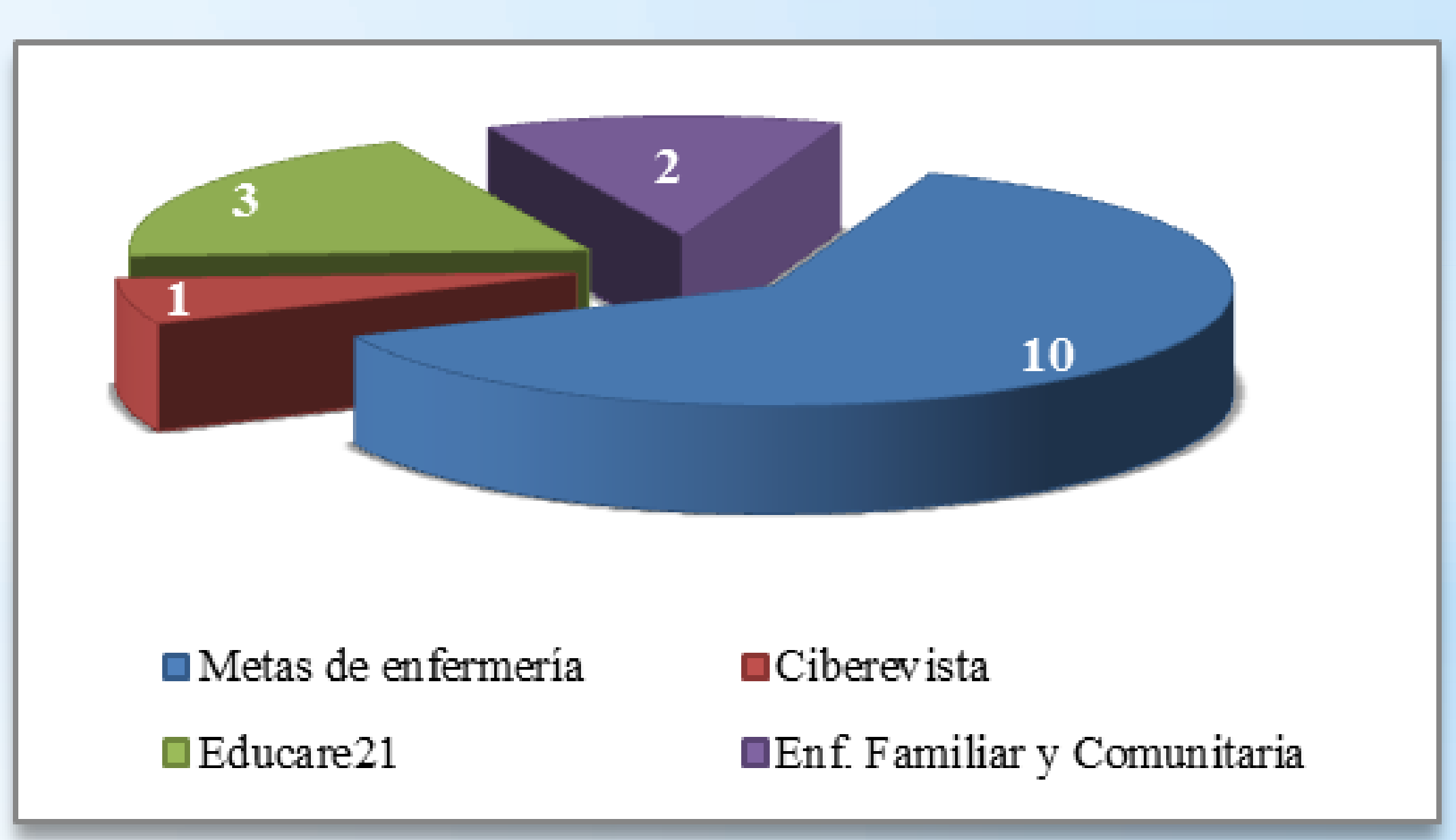

Ha habido un avance en cuanto a publicaciones específicas en la continuidad de cuidados, gestión de casos y relación de ayuda (encontrando un mayor número entre 2010-2011). Aun así existe poca bibliografía específica para estos términos.

El nuevo rol de la enfermera gestora de casos, garante de la continuidad de cuidados, redunda en una mayor satisfacción de los profesionales sanitarios y de los pacientes, así como en una contención de costes, mejora de los indicadores de calidad asistencial y los resultados de salud en general.

Establecer una relación clínica basada en una comunicación eficaz con el paciente y/o cuidadores, en la confianza, el respeto y la empatía entre el personal de enfermería y el paciente, es imprescindible para garantizar una atención integral y continuada. La gestión de casos ha demostrado tener una influencia positiva sobre el autocuidado y la adherencia al régimen terapéutico.

Es necesario que continúen las investigaciones que aborden de manera crítica y estandarizada la gestión de casos y la continuidad de cuidados para poder protocolizarlos e incluirlos en los planes actuales y futuros de cuidados.

\section{Agradecimientos}

Agradezco la inestimable colaboración en la revisión literaria que ha permitido la realización de este póster a:

Ramón Vicedo Cano

Elisa Marín Sánchez

Ángela Pérez Sánchez

Mํㅡㄹ Carmen Martínez González

Sin olvidar la ayuda de María Cabello Ganuza.

\section{Bibliografía}

Calero Romero MR (2012). Percepción del paciente sometido a trasplante de médula ósea sobre la continuidad de los cuidados. Educare21; 10(3).

Díaz Sánchez V et al. (2010). La construcción social del duelo en el aborto espontáneo. Metas de Enferm; 13(6): 25-32. Espina Boixo MA et al. (2011). Continuidad de cuidados desde el enfermero de emergencias hacia la enfermera de $\mathrm{Cl}$ de pacientes con SCACEST. Metas de Enferm; 14(2): 60-65.

Fernández Morenatti MA (2010). Experiencia de trabajo en un proceso de hospitalización domiciliaria. Metas de Enferm 13(1): 58-61.

Fontanet Cornudella G et al. (2010). La Enfermera virtual, un portal de educación y promoción para la salud. Metas de Enferm; 13(7): 50-54

García Pérez E (2009). La gestión de casos en la Clasificación de Intervenciones de Enfermería. Metas de Enferm; 12(4): 50-57

García Verde I et al. (2010). Problemas enfermeros en pacientes en situación terminal: caminando hacia la gestión de casos. Metas de Enferm; 13(3): 26-31.

Hanzeliková Pogrányivá A y Pomares Martínez D (2010). Plan de cuidados estandarizado: fractura de cadera en pacientes geriátricos. Educare21; $3(8)$.
Jiménez Fernández JC y Cerrillo Martín D (2010) Registros de Enfermería: un espejo del trabajo asistencial. Metas de Enferm; 13(6): 8-11.

Morales Asencio JM (2010). El liderazgo de la atención a personas con enfermedades crónicas complejas. RidEC; 3(2): 97 105

Padilla Just MN et al. (2011). Seguimiento telefónico enfermero a pacientes con insuficiencia cardíaca. Metas de Enferm; 14(3): 22-26.

Ramírez Sánchez A (2011). Síndrome de fatiga crónica en el adulto: atención enfermera. Educare21; 77.

Saavedra Llobregat MD et al. (2010) Cuidadores familiares. Aprendiendo a vivir. RIdEC; 3(2): 84-90.

Sanz Peces EM et al. (2010). Atención enfermera a pacientes con enfermedades neurodegenerativas en fase avanzada. Metas de Enferm; 13(9): 29-32.

Vázquez Calatayud M (2010). La vulnerabilidad y su relación con el cuidado. Metas de Enferm; 13(3): 72-74.

Vergara Santos S (2011). Implantación de plan de cuidados para pacientes pluripatológicos de la UGC de Medicina Interna-B del Hospital Universitario "Virgen Macarena". Ciberevista; 19. 